\title{
Use of cetuximab in combination with pulsed reduced dose-rate radiotherapy in a patient with recurrence of nasopharyngeal carcinoma in the neck
}

\author{
GUANG-HUI LI, BO ZHU, FAN YANG, CHUAN-KUN MA and DING-QIANG YANG \\ Institute for Cancer Research in People's Liberation Army, Xinqiao Hospital, \\ Third Military Medical University, Chongqing 400037, P.R. China
}

Received January 16, 2012; Accepted February 20, 2012

DOI: $10.3892 /$ etm.2012.506

\begin{abstract}
Reirradiation is a major therapeutic modality for patients with locally recurrent head and neck carcinoma. Due to normal tissue tolerances, reirradiation using conventional techniques has a narrow therapeutic ratio in the regional recurrence of nasopharyngeal carcinoma (NPC). Pulsed reduced dose-rate radiotherapy (PRDR), which delivers a series of 0.2 Gy pulses separated by 3 min intervals, is a new reirradiation technique. Head and neck carcinoma cells have high levels of epidermal growth factor receptor expression and cetuximab shows a clear benefit to locally advanced head and neck carcinoma. We report a 56-year-old male with a recurrent lesion of NPC in the neck following initial radical radiochemotherapy. The patient was retreated with PRDR and concurrent cetuximab. The total dose of PRDR was $70 \mathrm{~Gy}$, using 35 daily fractions of $2.0 \mathrm{~Gy}$. The recurrent lesion of this patient had a complete response with no apparent radiation-induced normal tissue complications. This is the first study concerning PRDR combined with cetuximab for the treatment of recurrent head and neck carcinoma following radiotherapy. The outcome of this patient reveals that treatment with PRDR and concurrent cetuximab is a promising therapeutic option for patients with recurrent head and neck carcinoma following radiotherapy.
\end{abstract}

\section{Introduction}

Nasopharyngeal carcinoma (NPC) is the most common type of head and neck cancer among the Southeastern Asian population. Radiotherapy is an important treatment approach for NPC (1). The recurrence of NPC following radiotherapy is a

Correspondence to: Dr Guang-hui $\mathrm{Li}$, Institute for Cancer Research in People's Liberation Army, Xinqiao Hospital, Third Military Medical University, Chongqing 400037, P.R. China

E-mail: liguanghui_2000@yahoo.com.cn

Key words: Cetuximab, pulsed reduced dose-rate radiotherapy, nasopharyngeal carcinoma, recurrence major modality of failure in patients with NPC. Approximately $20 \%$ of patients with NPC present with local failure following initial radiotherapy (2). Recurrence leads to a poor prognosis in patients with NPC since the sensitivity to radiotherapy is reduced in the recurrent tumor. Palliative therapeutic modalities include reirradiation, radioactive seed implantation, chemotherapy and surgical debulking for locally recurrent NPC. Reirradiation has gained wide acceptance and offers a chance of long-term tumor control for patients with local recurrence of NPC (3). However, the tolerance and toxicity of normal tissues experienced during reirradiation compared with the initial radiotherapy is a serious challenge.

A new technique of reirradiation, pulsed reduced dose-rate radiotherapy (PRDR), was developed for the local failure of tumors following radiotherapy. PRDR delivers a series of 0.2 Gy pulses separated by 3 min intervals. It has a high local control rate and is well-tolerated in patients with local recurrence of breast cancer or glioblastoma (4,5). Epidermal growth factor receptor (EGFR) is usually overexpressed in NPC (6). The overexpression of EGFR in squamous cell carcinoma of the head and neck is associated with a lower local control following radiotherapy (7). Cetuximab, an anti-EGFR monoclonal antibody, modulates apoptosis and enhances the effects of radiation and chemotherapy $(8,9)$. Therefore, in the present study a patient with a second neck recurrence of NPC received combined treatment of PRDR and cetuximab following two courses of radiotherapy and several cycles of chemotherapy.

\section{Case report}

A 56-year-old Asian male experienced a firm neck mass in September 2003. A computed tomography (CT) scan revealed an enhanced mass in the nasopharynx and several bilateral enhanced lymph nodes in the neck. A flexible fiberoptic nasopharyngoscope examination was performed and biopsies from the lesion of the nasopharynx were obtained. The biopsies were identified as poorly differentiated squamous cell carcinoma (Type II according to the WHO). Following a CT scan of the head/neck and thorax and ultrasound examination of the abdomen, the cancer of the patient was classified as stage $\mathrm{T} 2 \mathrm{~N} 2 \mathrm{M} 0$, based on clinical and radiological evaluation 

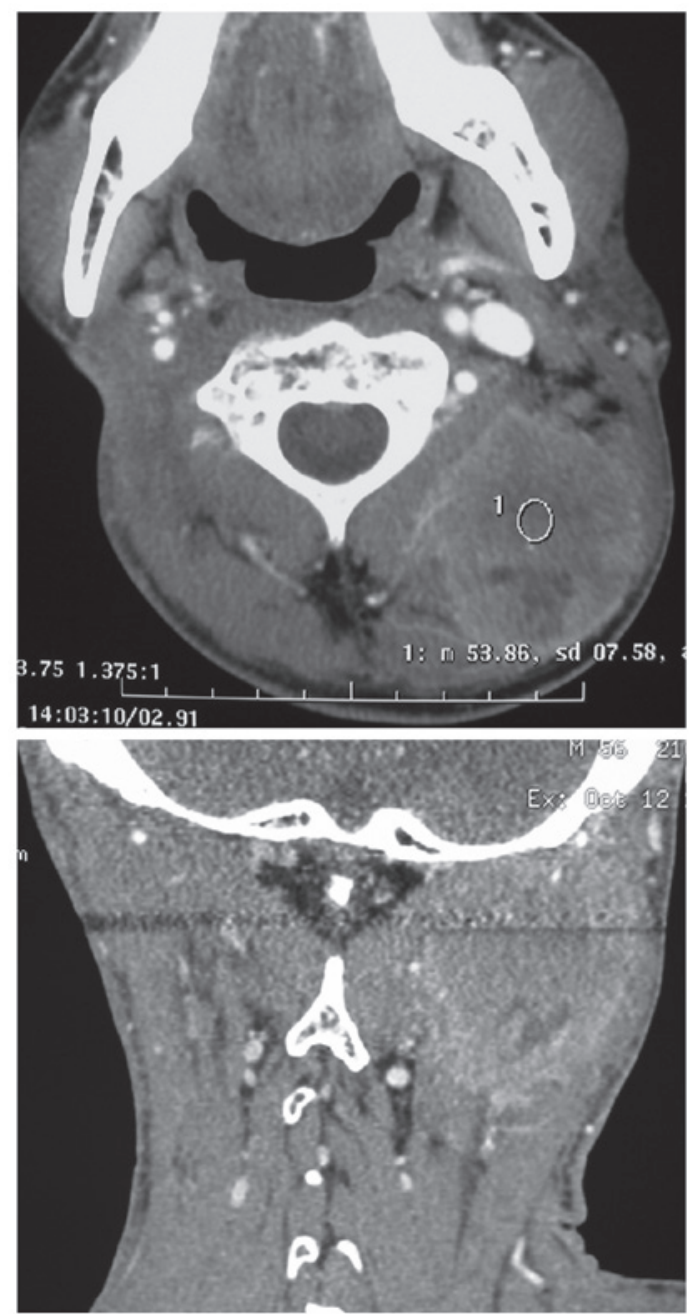

Figure 1. CT scan showing a large recurrent enhancing mass in the left neck of a patient with NPC following initial radiochemotherapy. CT, computed tomography; NPC, nasopharyngeal carcinoma.

and according to the TNM staging criteria (AJCC 2002) (10).

The patient received radical conventional external-beam radiotherapy (EBRT) with $8 \mathrm{MV}$ X-rays and 6-12 MeV electrons from a linear accelerator. Facial-cervical, preauricular and cervical tangent fields were applied. A total dose of $70 \mathrm{~Gy}$ was administered to the gross tumor targets and metastatic lymph node and $\geq 50$ Gy to the bilateral cervical lymphatics. EBRT was delivered with a daily fraction of $2 \mathrm{~Gy}$, five fractions per week. The patient was then followed up according to standard medical protocol. Endoscope and CT examinations revealed the disappearance of the gross tumor and metastatic lymph nodes at the first follow-up 1 month after radiation. A lymph node $1.5 \mathrm{~cm}$ in diameter was found on the right upper neck of the patient in October 2004. The patient refused to undergo a PET/CT or MRI scan or a biopsy of the lymph node. The patient also declined to treat this lymph node, which grew slowly. A contrast-enhanced CT scan revealed multiple bilateral metastatic lymph nodes in the neck of the patient in November 2009 and the greatest dimension of the mass in the right upper neck was $6 \mathrm{~cm}$. Three-dimensional conformal radiotherapy (3D-CRT) was performed to treat the recurrent metastatic lymph nodes in the neck. The dosage of
A

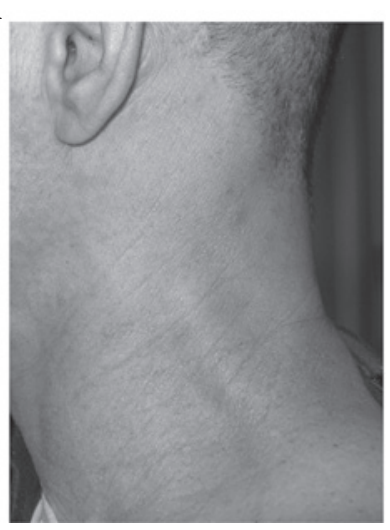

B

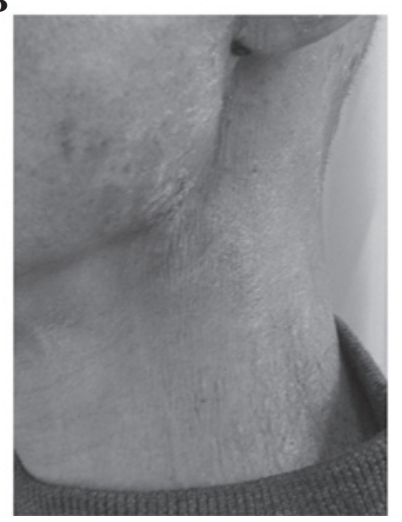

Figure 2. Radiation-induced acute skin toxicity of the left neck skin. (A) Prior to PRDR. (B) At the time of completed PRDR radiotherapy. PRDR, pulsed reduced dose-rate radiotherapy.

clinic target volume (CTV) was 60 Gy. The reliquus metastatic lymph nodes in the right neck were surgically resected following reirradiation therapy. Adjuvant chemotherapy was initiated within 2 weeks of surgery for 4 cycles. The chemotherapy regimen consisted of paclitaxel, $135 \mathrm{mg} / \mathrm{m}^{2}$ on day 1 , and cisplatin, $30 \mathrm{mg} / \mathrm{m}^{2} /$ day on days $1-3$ of a 21 -day treatment cycle.

In May 2010, a 1.5x $2 \mathrm{~cm}$ mass in the left upper neck was found by the patient. A contrast-enhanced CT scan of the neck revealed that the mass was a recurrent metastatic lymph node. No other metastastic lesion was found following a CT scan of the head/neck and thorax and ultrasound examination of the abdomen. The patient declined to undergo surgical management and received chemotherapy with cisplatin $\left(90 \mathrm{mg} / \mathrm{m}^{2}\right.$ on day 1) plus fluorouracil (500 mg/m²/day for 5 days) for 2 cycles. The mass increased slowly in size and paclitaxel $\left(135 \mathrm{mg} / \mathrm{m}^{2}\right.$ on day 1) and cisplatin (30 $\mathrm{mg} / \mathrm{m}^{2} /$ day on day 1-3) were used for 2 cycles. The lesion progressed and the greatest dimension of the mass was $5.5 \mathrm{~cm}$ (Fig. 1). The clinical consensus was to proceed with PRDR and concurrent cetuximab following an evaluation of the medical oncology and the choice of the patient. PRDR was performed using 3D-CRT and each fraction was delivered with 0.2 Gy pulses separated by 3 min intervals, creating a dose-rate of $0.0667 \mathrm{~Gy} / \mathrm{min}(7,8,18)$. The patient received a total dose of 70 Gy using 35 daily fractions of 2.0 Gy from 15 October 2010 to 2 December 2010. Cetuximab was administered at an initial dose of $400 \mathrm{mg} / \mathrm{m}^{2}$ and was subsequently administered at a weekly dose of $250 \mathrm{mg} / \mathrm{m}^{2}$ concurrently with radiotherapy (11).

The side effects of reirradiation and cetuximab were well-tolerated by the patient. At the third week of radiotherapy, papulo-pustular skin lesions developed in the face of the patient. The patient experienced grade 1 skin toxicity in response to cetuximab and grade 2 pharyngeal mucositis during radiotherapy. Notably, the patient experienced only grade 1 acute skin toxicity induced by irradiation in the reirradiated field and no significant late toxicity (Fig. 2). In February 2011, a contrast-enhanced CT scan of the neck of the patient revealed the complete response of the recurrent lymph node in the left neck following treatment with PRDR plus cetuximab (Fig. 3). 

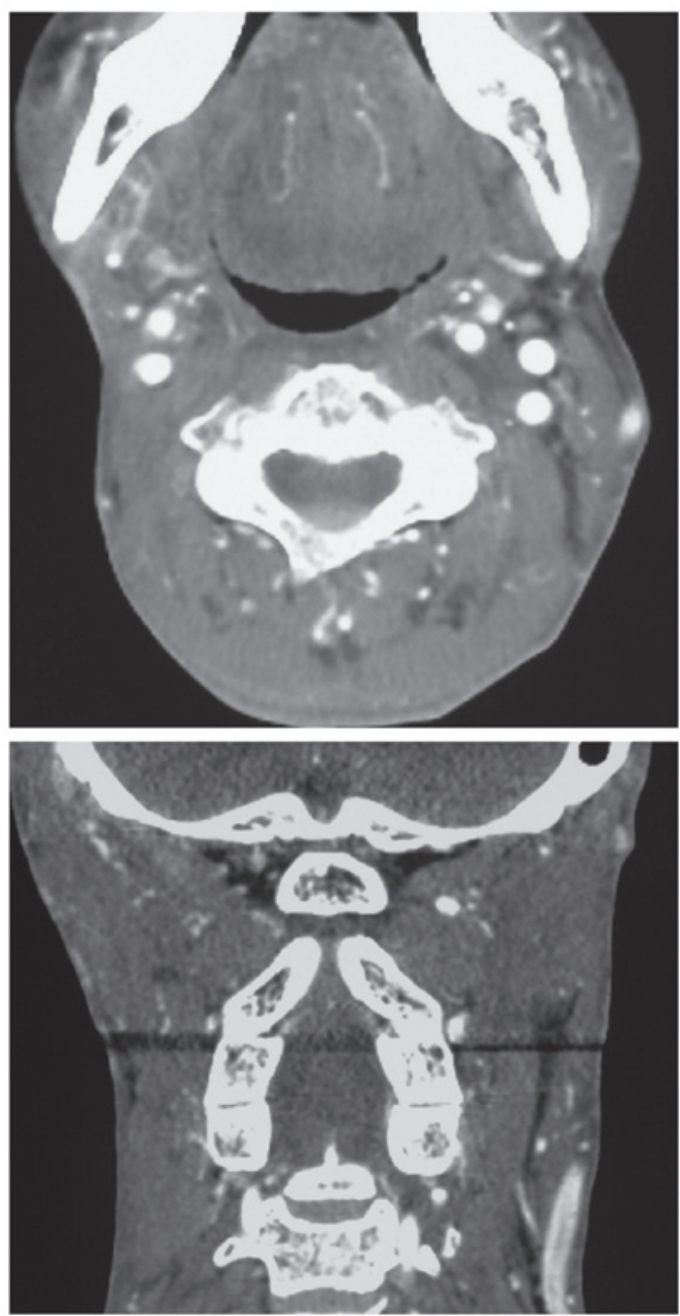

Figure 3. CT images with contrast ( 2 months following the completion of PRDR radiation with concurrent cetuximab). The recurrent lesion regressed. $\mathrm{CT}$, computed tomography; PRDR, pulsed reduced dose-rate radiotherapy.

\section{Discussion}

Curative approaches to recurrent NPC include surgery with or without radiotherapy and definitive radiation-based therapy. Recurrent disease is often not resectable, or patients decline surgical resection due to concerns about quality of life. Reirradiation is an important approach for the treatment of patients with recurrent head and neck carcinoma. However, reirradiation is difficult due to normal tissue tolerances. Normal tissues that receive reirradiation may experience more severe acute and late radiation complications compared with the initial course. It is difficult to achieve the correct balance between tumor control and the severe toxicity of normal tissue. Reirradiation using conventional techniques often has modest palliative and survival benefits.

A new irradiation technique, PRDR, was developed to treat recurrent malignant lesions. It appears to be a highly effective modality on irradiated recurrences and a low toxicity method to reirradiate normal tissues. Due to the different repair capacities of normal tissues and tumor cells, below standard dose-rates are preferable to protect normal tissue, while PRDR produced almost identical toxicity to cancer cells as conventional radio- therapy. The mechanism by which PRDR kills tumor cells may be low dose hyperradiosensitivity (LDHRS). LDHRS, which is increased radiosensitivity to doses $<0.3-0.5 \mathrm{~Gy}$, has been demonstrated in numerous tumor cells $(12,13)$. On the other hand, the reduced dose-rate and a fixed time interval reduce toxicity and improve the sublethal damage repair of normal tissue. It allows normal tissues to repair during each sub-fraction and is an effective method with low toxicity to treat recurrent breast cancer and glioblastoma $(4,5)$.

As squamous cell carcinoma of the head and neck commonly has a high level of EGFR expression, treatment with cetuximab shows the clearest benefit to locally advanced head and neck cancer compared with radiotherapy alone when it is combined with radiotherapy (14). We treated a patient with recurrent NPC with PRDR and concurrent cetuximab. The treatment of the recurrent neck lesion in this patient achieved complete regional control and low toxicity to normal tissue around the lesion. The combination of radiation and concurrent cetuximab has been reported to enhance skin toxicity compared with radiotherapy alone in patients with head and neck cancer $(15,16)$. However, only CTC grade 1 radiodermatitis occurred in this patient. Certain studies have reported a lack of cetuximab-induced skin rash in the previously radiated field. The mechanism of this phenomenon is uncertain $(17,18)$. It may be related to microvascular injury which reduces the delivery of cetuximab, a reduced number of EGFR-expressing cells or a reduced receptor sensitivity following radiation.

In conclusion, the treatment of PRDR with concurrent cetuximab is well-tolerated and is a promising therapeutic option for patients with recurrent head and neck carcinoma following radiotherapy, although the mechanism by which it functions remains unclear.

\section{References}

1. Shen C, Gao Y, Xu T, et al: Carcinoma of the nasopharynx in young patients: a single institution experience. Clin Oncol ( $R$ Coll Radiol) 21: 617-622, 2009.

2. Yi JL, Gao L, Huang XD, et al: Nasopharyngeal carcinoma treated by radical radiotherapy alone: ten-year experience of a single institution. Int J Radiat Oncol Biol Phys 65: 161-168, 2006.

3. Sher DJ, Haddad RI, Norris CM, et al: Efficacy and toxicity of reirradiation using intensity-modulated radiotherapy for recurrent or second primary head and neck cancer. Cancer 116: 4761-4768, 2010

4. Adkison JB, Tomé W, Seo S, et al: Reirradiation of large-volume recurrent glioma with pulsed reduced-dose-rate radiotherapy. Int J Radiat Oncol Biol Phys 79: 835-841, 2011.

5. Richards GM, Tomé WA, Robins HI, et al: Pulsed reduced dose-rate radiotherapy: a novel locoregional retreatment strategy for breast cancer recurrence in the previously irradiated chest wall, axilla, or supraclavicular region. Breast Cancer Res Treat 114: 307-313, 2009.

6. Chung CH, Ely K, McGavran L, et al: Increased epidermal growth factor receptor gene copy number is associated with poor prognosis in head and neck squamous cell carcinomas. J Clin Oncol 24: 4170-4176, 2006.

7. Zimmermann M, Zouhair A, Azria D and Ozsahin M: The epidermal growth factor recepter (EGFR) in head and neck cancer: its role and treatment implications. Radiat Oncol 1: 11-16, 2006.

8. Bonner JA, Harari PM, Giralt J, et al: Radiotherapy plus cetuximab for squamous-cell carcinoma of the head and neck. N Engl J Med 354: 567-578, 2006.

9. Sharafinski ME, Ferris RL, Ferrone S and Grandis JR: Epidermal growth factor receptor targeted therapy of squamous cell carcinoma of the head and neck. Head Neck 32: 1412-1421, 2010. 
10. Greene FL,wPage DL, Fleming ID, Fritz A, Balch CM, Haller DG and Morros M (eds): AJCC Cancer Staging Manual. 6th ed. Springer-Verlag, New York, 2002.

11. Mendelsohn J and Baselga J: Status of epidermal growth factor receptor antagonists in the biology and treatment of cancer. J Clin Oncol 21: 2787-2799, 2003.

12. Harney J, Short SC, Shah N, et al: Low dose hyperradiosensitivity in metastatic tumors. Int J Radiat Oncol Biol Phys 59: 1190-1195, 2004.

13. Lin PS and Wu A: Not all 2 Gray radiation prescriptions are equivalent: cytotoxic effect depends on delivery sequences of partial fractionated doses. Int J Radiat Oncol Biol Phys 63: 536-544, 2005

14. Specenier P and Vermorken JB: Cetuximab in the treatment of squamous cell carcinoma of the head and neck. Expert Rev Anticancer Ther 11: 511-524, 2011.
15. Walsh L, Gillham C, Dunne M, et al: Toxicity of cetuximab versus cisplatin concurrent with radiotherapy in locally advanced head and neck squamous cell cancer (LAHNSCC). Radiother Oncol 98: 38-41, 2011

16. Pryor DI, Porceddu SV, Burmeister BH, et al: Enhanced toxicity with concurrent cetuximab and radiotherapy in head and neck cancer. Radiother Oncol 90: 172-176, 2009.

17. Bossi P, Liberatoscioli C, Bergamini C, et al: Previously irradiated areas spared from skin toxicity induced by cetuximab in six patients: implications for the administration of EGFR inhibitors in previously irradiated patients. Ann Oncol 18: 601-602, 2007.

18. Kanakamedala MR, Packianathan S and Vijayakumar S: Lack of cetuximab induced skin toxicity in a previously irradiated field: case report and review of the literature. Radial Oncol 5: 38-41, 2010. 